\title{
Analysis of Fungal Diversity in Ready-to-Eat Pizza and Effectiveness of Pulsed Ultraviolet-Light Treatment for Inactivation of Mold on Agar Surface
}

Tina M Beigi ${ }^{1}$, Michael O Ngadi*, Devin B Holman² and Martin R Chénier ${ }^{2,3}$

${ }^{1}$ Department of Bio resource Engineering, McGill University, Ste-Anne-de-Bellevue, QC, Canada

${ }^{2}$ Department of Animal Science, McGill University, Ste-Anne-de-Bellevue, QC, Canada

${ }^{3}$ Department of Food Science and Agricultural Chemistry, McGill University, Ste-Anne-de-Bellevue, QC, Canada

\begin{abstract}
Fungal contamination is a significant issue in the food production industry; therefore, identification and characterization of food spoilage fungi would allow for early intervention to limit the amount of fungal contamination, particularly in cereal-based industries. In the present study, culture-dependent and culture-independent methods were applied to study the microbiota of ready-to-eat pizza. The study was pursued by evaluating the effectiveness of a broad-spectrum pulsed ultraviolet light for the decontamination of Penicillium roqueforti (a dominant spoilage mold in bakery products) on the surface of solid agar as a representative of a flat food surface. The average population of mesophilic aerobic bacteria (MAB), mesophilic anaerobic bacteria (MANB), lactic acid bacteria (LAB), molds and yeasts $(M+Y)$ on naturally spoiled pre-cooked pizza were $6.7 \pm 0.5$, less than $2.3,2.8 \pm 0.6$ and $5.4 \pm 0.4 \log 10 \mathrm{CFU} \mathrm{g}^{-1}$, respectively. Cloning and sequencing identified at least 5 genera and species of fungi (Penicillium spp., Saccharomyces spp., Rhodotorula mucilaginosa, Monascus fuliginosus, Galactomyces geotrichum) from 2 phyla (Ascomycota and Basidiomycota). Pulsed light process parameters evaluated were treatment time $(1,3,5,7$ and $10 \mathrm{~min})$ and voltage input $(500,750$ and 1,000 V) at $5 \mathrm{~cm}$ distance from the pulsed UV-light. An increase of the input voltage of the lamps and of the duration of the treatment resulted in a higher inactivation of $P$. roqueforti. The population of $P$. roqueforti was reduced after 10

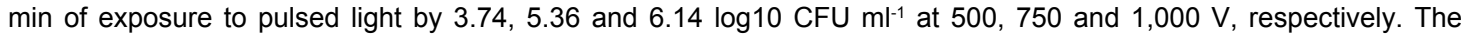
inactivation kinetics was best described by the Weibull model (2 parameters) with the smallest root mean squared error (RMSE) and $R^{2} \geq 0.92$. The results of this work show that pulsed light is a promising technique for fungi elimination or decontamination in the bakery industry.
\end{abstract}

Keywords: Cloning; Food safety; Kinetics; Mold; Pulsed UV-light; Ready-to-eat pizza

\section{Introduction}

Bakery products remain an important component of a balanced diet and pizza in particular continues to be one of the most widely consumed fast foods in the world. Physical, chemical and microbial spoilage are of concern in most cereal-based products including pizza. Spoilage caused by fungi is considered to be the greatest factor in limiting shelf-life and the major cause of economic losses in the bakery industry. Ready-to-eat food products have become increasingly popular due to their convenience and improved flavor.

Ready-to-eat tomato pizza consists of oven baked white bread topped with tomato sauce and can be consumed without additional cooking. In food products that have been pre-cooked such as ready-toeat pizza dough, fungi may be inactivated during baking, however, the food product may become contaminated again after cooking. Two types of fungi that are frequently found in ready-to-eat pre-cooked pizza dough belong to the Penicillium and Aspergillus genera [1]. Recently, molecular methods have provided another means for the detection, quantification, identification, and characterization of microorganisms in food products. These methods are now considered an indispensable tool that permits a more accurate description of the microbial ecology of food than culture-dependent methods alone [2,3]. Polymerase chain reaction (PCR) using domain or taxon-specific probes in combination with separation techniques such as DGGE and molecular cloning have revolutionized our understanding of the composition of food microbiota [4].

Pulsed ultraviolet-light is a unique emerging technology used mostly for surface decontamination. This non-thermal sterilization technology uses intense flashes of broad-spectrum radiation ranging from UV light $(200 \mathrm{~nm})$ to infrared radiation $(1,000 \mathrm{~nm})$ [5]. This novel technique is capable of reducing microbial populations of both vegetative cells and spores of bacteria, molds and yeasts [6]. Pulsed UV-light has been investigated as a means of extending the shelf-life of baked goods, seafood, meat products, and fruits and vegetables [7]. The ability of pulsed UV-light to kill fungal spores is well documented and studies have shown a 60-99\% reduction of viable spores of Aspergillus flavus, Aspergillus niger, Penicillium corylophilum and Eurotium rubrum after only a $15 \mathrm{~s}$ exposure to pulsed UV-light [8]. Pulsed UVlight has also been successfully used to significantly reduce suspensions of Botrytis cinerea and Monilia fructigena conidia and to inactivate Saccharomyces cerevisiae in non-alcoholic beer $[9,10]$. In a study by Jun et al. [11] on corn meal, a $100 \mathrm{~s}$ treatment time, $3 \mathrm{~cm}$ of distance from the UV strobe with $3,800 \mathrm{~V}$ input gave a $4.93 \mathrm{CFU}$ g- $1 \log 10$ reduction to the spore of $A$. niger.

In order to further understand the potential of pulsed UV light on mold decontamination, the response of Penicillium roqueforti to pulsed UV-light treatment need to be understood through modeling.

*Corresponding author: Michael O Ngadi, Department of Bioresource Engineering, McGill University, 21,111 Lakeshore Road, Ste-Anne-de-Bellevue, Quebec, Canada, H9X 3V9, Tel: +1-514-398-7923; Fax: +1-514-398-7990; E-mail michael.ngadi@mcgill.ca

Received April 20, 2015; Accepted May 18, 2015; Published May 22, 2015

Citation:BeigiTM,NgadiMO, HolmanDB, ChénierMR(2015)PectinMethylesterases: A Review. J Bioprocess Biotech 5: 228 doi:10.4172/22159821.1000228

Copyright: @ 2015 Beigi TM et al. This is an open-access article distributed under the terms of the Creative Commons Attribution License, which permits unrestricted use, distribution, and reproduction in any medium, provided the original author and source are credited. 
The inactivation of microorganisms via UV light has been shown to follow first-order kinetics in solution [12], but it has also been reported to display a sigmoidal shape with a shoulder and/or a tail [13]. The aim of the present study is to analyze the abundance and composition of the microbial community found on ready-eat-to-eat pizza, with a focus on molds, and to determine the proper pulsed UV treatment necessary to construct inactivation model kinetics of a spoilage mold.

\section{Materials and Methods}

\section{Sampling}

Pre-cooked pizza samples that had been naturally contaminated with spoilage molds were obtained from a pizza-manufacturing company in the city of Montreal, QC, Canada. The samples consisted of baked white bread covered with tomato sauce. Samples for enumeration and identification purposes were collected at two different time intervals with 10 samples in June and 10 in September of 2013. Samples collected in June were used for the microbial analysis and those collected in September were used in order to create the $18 \mathrm{~S}$ clone library.

\section{Preparation of pizza samples for microbial enumerations}

All of the spoiled pizzas were aseptically removed from their individual package and weighed. A $25 \mathrm{~g}$ amount of each sample was then placed in a sterile stomacher bag containing $225 \mathrm{ml}$ of buffered peptone water (Don Whitley Scientific, WY, UK) (in the proportion with pizza samples at 10:1). The mixture was then blended in a Stomacher 400 Lab Blender (Seward medical, London, UK) for 2 min or until homogenized. From the resulting mixture, serial decimal dilutions (10-1 to 10-10) were conducted in buffered peptone water and the dilutions were spread-plated in triplicate onto appropriate media, as described below.

\section{Microbial enumerations}

To determine the total population of mesophilic aerobic bacteria (MAB) and mesophilic anaerobic bacteria (MANB), Petri plates containing Plate Count Agar (PCA, Oxoid, Hampshire, UK) were prepared. Plates were incubated at $35^{\circ} \mathrm{C}$ for $48 \mathrm{~h}$ under aerobic (MAB) or anaerobic (MANB) conditions. Anaerobic incubation was carried out in a Forma anaerobic system, model 1025/1029 (Thermo Scientific, Marietta, Ohio, USA) [13]. In order to enumerate populations of lactic acid bacteria (LAB), MRS Agar and M17 agar (Oxoid, Hampshire, UK) were prepared with a lactose solution $(5 \% \mathrm{v} / \mathrm{v})$ according to Terzaghi and Sandine [14]. Plates were incubated at $37^{\circ} \mathrm{C}$ for $48-72 \mathrm{~h}$. Molds and yeasts $(\mathrm{M}+\mathrm{Y})$ were enumerated on Potato Dextrose Agar (PDA, Oxoid, acidified to $\mathrm{pH} 3.5$ with a solution of $10 \%$ of tartaric acid). Plates were incubated at $24^{\circ} \mathrm{C}$ for 3-5 days. Colonies were counted with the aid of a Quebec Colony Counter (Reichert Technologies, Depew, NY, USA) and results expressed in terms of CFU g ${ }^{-1}$ food product.

\section{DNA extraction}

Packaged precooked pizza samples with signs of spoilage were aseptically opened and one gram of each sample (taken from a spoiled portion of the sample) was weighed and placed into a sterile stomacher bag. Sterile $0.1 \%$ peptone water $(1 \mathrm{ml})$ was added to each sample and the contents homogenized in a Stomacher $400 \mathrm{Lab}$ Blender (Seward Medical, London, UK) for $2 \mathrm{~min}$. Total DNA was subsequently extracted using a Power Food Microbial DNA Isolation Kit (MO BIO Laboratories, Inc., Carlsbad, CA, USA) according to manufacturer's instructions. The concentration of eluted DNA was measured using a NanoDrop 2000 Spectrophotometer (Thermo Fisher Scientific, Wilmington, DE, USA). DNA extracts were stored at $-20^{\circ} \mathrm{C}$ until analysis as recommended by manufacturer. Although DNA extraction was from each of the 10 samples, insufficient DNA was extracted from two samples leaving a total of 8 samples used for fungal $18 \mathrm{~S}$ sequencing.

\section{PCR amplification of fungal $18 S$ rDNA}

Fungal (molds and yeasts) 18S rRNA genes were amplified from extracted DNA from spoiled pizza samples using primers NS1-F and NS6-F (Integrated DNA Technologies, Toronto, ON, Canada; Table 1). All PCR reactions were prepared in a dedicated PCR laminar flow cabinet (Esco Technologies Inc., PA, USA). PCR reaction mixtures and thermal cycling conditions were as described in $\mathrm{Wu}$ and Blomquist [15]. Briefly, each PCR reaction consisted of: 1×buffer (Denville Scientific, Saint-Laurent, QC, Canada), $500 \mu \mathrm{M}$ of each primer, 1.5 $\mathrm{U}$ of Hot Start Taq DNA polymerase (Denville Scientific, SaintLaurent, QC, Canada), $200 \mathrm{mM}$ of each dNTP (Bioshop Canada Inc., Burlington, ON, Canada), 1-5 ng of template DNA extracted from the spoiled samples, and molecular grade water (Sigma-Aldrich, Oakville, ON, Canada) in a total volume of $50 \mu \mathrm{l}$. PCR amplifications were conducted using a Veriti Thermocycler (Applied Biosystems, Foster City, CA, USA) with the following temperature cycling profile: initial denaturation at $94^{\circ} \mathrm{C}$ for $3 \mathrm{~min}$, followed by 35 cycles of denaturation at $94^{\circ} \mathrm{C}$ for $45 \mathrm{sec}$, annealing at $58^{\circ} \mathrm{C}$ for $50 \mathrm{sec}$, and extension at $72^{\circ} \mathrm{C}$ for $90 \mathrm{sec}$, followed by $5 \mathrm{~min}$ at $72^{\circ} \mathrm{C}$ for the final extension. A positive control with DNA extracted from Rhizoctonia solani and a negative control consisting of the reaction mixture without DNA were used in each PCR run. PCR products were run at $75 \mathrm{~V}$ for $85 \mathrm{~min}$ on a $1.5 \%$ agarose gel stained with ethidium bromide and visualized using a Red AlphaImager (Protein simple, Santa Clara, CA, USA). A Gene Ruler 100-bp DNA ladder (Thermo Fisher Scientific Inc.) was used to estimate the size of the products.

\section{Cloning and sequencing of fungal $18 S$ rDNA PCR products}

PCR products were purified using a SpinSmart PCR purification kit (Denville Scientific Inc., Metuchen, NJ, USA) and cloned into a pGEM-T Easy vector (Promega, Madison WI, USA) according to manufacturer's protocol. Four to six white colonies (positive transformants) for each sample were selected and screened by PCR for the presence of an insert using primers M13-F and M13-R (Table 1). Each PCR reaction contained $1 \times$ PCR buffer, $200 \mathrm{nM}$ of each primer (Integrated DNA Technologies, Toronto, ON, Canada), $80 \mu \mathrm{M}$ dNTPs (Bioshop Canada Inc., Burlington, ON, Canada), 2 U Hot-Start Taq DNA polymerase (Denville Scientific, Saint-Laurent, QC, Canada), and molecular grade water (Sigma-Aldrich, Oakville, ON, Canada) in a total volume of 50 $\mu \mathrm{l}$. The thermal cycling conditions were as follows: initial denaturation at $95^{\circ} \mathrm{C}$ for $3 \mathrm{~min}$, followed by 35 cycles of denaturation at $94^{\circ} \mathrm{C}$ for 30 sec, annealing at $57^{\circ} \mathrm{C}$ for $30 \mathrm{sec}$ and extension at $72^{\circ} \mathrm{C}$ for $1 \mathrm{~min}$, and then $10 \mathrm{~min}$ at $72^{\circ} \mathrm{C}$ for the final extension. PCR products were verified for the correct insert length on a 1.5\% agarose gel.

PCR products containing the desired insert were selected for sequencing. In total, 28 clones were sequenced from 8 samples. Sequencing was carried out at the McGill University and Genome Québec Innovation Centre (Montreal, QC, Canada) using a $3730 \mathrm{xl}$ DNA Analyzer system (Applied Biosystems, Carlsbad, CA, USA). Sequences were checked for putative chimeras using DECIPHER [16] and chimeras were removed before comparing sequences with those in the Ribosomal Database project (RDP; http://rdp.cme.msu.edu) and BLASTn (http://blast.ncbi.nlm.nih. gov). Sequences were submitted to the GenBank database under accession numbers KJ958135 to KJ958162. 


\begin{tabular}{|c|c|c|c|c|}
\hline Primer name $^{\star}$ & Sequence $\left[5^{\prime} \rightarrow 3^{\prime}\right]$ & Target & Amplicon size [bp] & References \\
\hline NS1-F & GTAGTCATATGCTTGTCTC & Universal for fungi & 1400 & [42] \\
\hline NS6-R & GCATCACAGACCTGTTATTGCCTC & Universal for fungi & 1400 & [42] \\
\hline M13-F & GTAAAACGACGGCCAG & Plasmid linker region & 1663 & [24] \\
\hline M13-R & CAGGAAACAGCTATGAC & Plasmid linker region & 1663 & [24] \\
\hline
\end{tabular}

*Primer set NS1-NS6 was used for the generation of PCR products for TA cloning. Primer set M13 was used for the sequencing of PCR products cloned with the Easy T vector system.

Table 1: Oligonucleotide primers used in this study.

\section{Preparation of inoculum for pulsed UV light experiments}

As Penicillium spp. were identified using DNA sequencing, Penicillium roqueforti ATCC 10010 (Cedarlane Corporation, Burlington, Ontario, Canada) was used throughout pulsed UV light experiments. P. roqueforti was grown in Potato Dextrose Broth (PDB, Difco, Spark, MD, USA) with shaking at $180 \mathrm{rpm}$ at $24^{\circ} \mathrm{C}$ for 4 to 7 days. Cultures were maintained by spreading $0.1 \mathrm{ml}$ of liquid culture on PDA. After incubating the PDA plates at $24^{\circ} \mathrm{C}$ for 7 days, the spores were harvested by adding $5 \mathrm{ml}$ of Tween 80 (Acros Organic, New Jersey, USA) onto the surface of PDA, and then filtered by using 4 layers of cheese cloth. The resulting inoculum solution had $2.74 \times 10^{10}$ CFU ml ${ }^{-1}$ and this cell suspension was serially diluted up to $1: 100,000$. An inoculum of $0.1 \mathrm{ml}$ of each dilution (1:100, 1:1,000 and 1:100,000) was spread in triplicates on PDA plates.

\section{UV inactivation of $P$. roqueforti}

The pulsed-UV apparatus consists of a metal housing surrounding a treatment chamber made of polished stainless steel $(20 \mathrm{~cm}$ wide $\times 14$ $\mathrm{cm}$ deep $\times 12 \mathrm{~cm}$ high) and equipped with an upper Xenon lamp. PDA plates inoculated with $P$. roqueforti spores were positioned on the tray at $5 \mathrm{~cm}$ from the UV lamp in the treatment chamber. At this distance, the effect of treatment time $(1,3,5,7$ and $10 \mathrm{~min})$ and input voltages of 500, 750 and $1,000 \mathrm{~V}$ were investigated. Following UV treatment, all Petri plates were incubated for 4 days in the dark at $24^{\circ} \mathrm{C}$ and the impact of UV treatment on the inactivation of $P$. roqueforti was determined by enumerating the colonies on each PDA plate. The $\log 10$ reduction was calculated by subtracting the $\log 10$ value of the control from that of treated sample.

\section{Development of log-linear model}

Traditionally, microbial inactivation resulting from application of both thermal and non-thermal processes has been described by the log-linear equation [17]. This model (Equation 1) follows the rules of first-order inactivation kinetics to describe microorganism destruction [18].

$$
\log \left(\frac{N}{N_{o}}\right)=-k t
$$

Where $\mathrm{N}$ is the number of microorganisms at time $\mathrm{t}\left(\mathrm{CFU} \mathrm{ml}{ }^{-1}\right), \mathrm{N} 0$ is the initial number of microorganisms $\left(\mathrm{CFU} \mathrm{ml}{ }^{-1}\right), t$ is the treatment time (sec or min), $\mathrm{k}$ is the first-order extinction coefficient $\left(\mathrm{s}^{-1}\right.$ or min 1). From this equation, the classic " $D$-value" or the time necessary for a $1 \log 10$ reduction can be calculated as the reciprocal of the firstorder rate constant. The log-linear equation is simply appropriate for linear inactivation curves and research findings suggest that many inactivation curves are non-linear [17].

\section{Weibull model}

The second model used in the current study is the Weibull model (Equation 2) which is offered as a simple model for the description of microbial inactivation by thermal and non-thermal treatment methods [18]. In the case of UV-light treatment, inactivation curves are generally sigmoidal and exhibit concavity or convexity behaviors through downwards or upwards as a function of inactivation time or UV dose [19]. The Weibull model usually describes this behavior accurately. This model is composed of two parameters ( $\alpha$ and $\beta$ ) given in the following correlation $[17,18]$ :

$$
\log \left(\frac{N}{N_{o}}\right)=-\frac{1}{2.303}\left(\frac{t}{\alpha}\right)^{\beta}
$$

Where $\mathrm{N}$ is the number of microorganisms $\left(\mathrm{CFU} \mathrm{ml}{ }^{-1}\right), \mathrm{N}_{0}$ is the initial number of microorganisms $\left(\mathrm{CFU} \mathrm{ml}{ }^{-1}\right), \mathrm{t}$ is the treatment time (sec or min), $\alpha$ is the characteristic time (s or min) and $\beta$ is the shape parameter (unit less). Many survival curves exhibit concavity, either downwards or upwards and the $\beta$ parameter is used to describe this concavity. If $\beta<1$, the curve displays upward concavity. This can be a sign of stress adaptation of surviving microorganism. If $\beta>1$, the curve displays downward concavity that shows considerable damages of pulsed UV-light in the cells. In addition, by using $\alpha$ and $\beta$, reliable life (tR) (indicating the time necessary for $90 \%$ reduction in the number of microorganism) can be calculated [18]. This parameter corresponds to the D-value for the first log reduction (Equation 3 ).

$$
t_{R}=\alpha(2.303)^{\frac{1}{\beta}}
$$

Where $\alpha$ is a characteristic time and $\beta$ is the shape parameter.

\section{Statistical analysis}

Analysis of variance was performed using PROC GLM in SAS (SAS Inst., Inc., Cary, NC, USA) to compare the effects of different applied pulsed-light intensity (voltage input) and treatment time (number of pulses). All treatments were done in triplicate and results were considered significant at the $\alpha=0.05$ level.

\section{Results and Discussion}

\section{Enumeration of microbial populations}

In this work, the microbial diversity of 10 spoiled precooked pizza samples was studied. The samples were examined for the presence of mesophilic aerobic bacteria (MAB), mesophilic anaerobic bacteria (MANB), lactic acid bacteria (LAB), molds and yeasts $\left(\mathrm{M}+\mathrm{Y}\right.$ ) (Table 2 ). MAB counts varied from $6.5 \times 10^{5}$ (sample 8) to $2.2 \times 10^{7}$ (sample 5) $\mathrm{CFU} \mathrm{g}{ }^{-1}$ pizza product, with 4 samples having concentrations of approximately $10^{6} \mathrm{CFU} \mathrm{g}{ }^{-1}$ pizza product. MANB counts ranged from less than 101 to $2.7 \times 10^{2}$ (sample 5) CFU $\mathrm{g}^{-1}$ pizza product. These numbers are considerably lower than the MANB counts of $1.6 \times 10^{4} \mathrm{CFU}$ g reported by Rodriguez et al. [20] in study of the shelf life of pre-baked pizza dough. In all precooked ready-to-eat pizza samples, counts of LAB ranged between $1.2 \times 10^{2}$ (samples 3 and 4 ) and $1.6 \times 10^{4}$ (sample 7) CFU $\mathrm{g}^{-1}$. This is in agreement with a LAB concentration of $10 \times 10^{4} \mathrm{CFU}$ $\mathrm{g}^{-1}$ reported by Rodriguez et al. [20] in prepackaged pizza dough. 


\begin{tabular}{|c|c|c|c|c|}
\hline \multirow{2}{*}{ Samples } & \multicolumn{4}{|c|}{ Microbial populations $\left[\log _{10} \text { CFU }^{-1}\right]^{*}$} \\
\hline 1 & MAB & MANB & LAB & Y+M \\
\hline 2 & 6.8 & 1.6 & 2.5 & 5.3 \\
\hline 3 & 7.1 & $<1$ & 3.0 & 5.2 \\
\hline 4 & 6.1 & $<1$ & 2.1 & 4.9 \\
\hline 5 & 6.0 & 1.8 & 2.1 & 4.6 \\
\hline 6 & 7.4 & 2.4 & 2.9 & 5.2 \\
\hline 7 & 5.9 & $<1$ & 2.1 & 5.4 \\
\hline 8 & 6.8 & $<1$ & 4.2 & 5.6 \\
\hline 9 & 5.8 & $<1$ & 2.9 & 6.0 \\
\hline 10 & 6.5 & 1.8 & 3.0 & 5.9 \\
\hline Average & 7.2 & 1.2 & 2.7 & 5.8 \\
\hline
\end{tabular}

"Mean values of the CFU g-1 for each sample, analyzed in triplicate, are reported Refer to materials and methods for media specifications and incubation conditions. MAB: Mesophilic Aerobic Bacteria; MANB: Mesophilic Anaerobic Bacteria; LAB: Lactic Acid Bacteria; Y+M: Yeasts and Molds; $\log _{10}$ : Logarithm to the base 10 ; CFU: Colony Forming Units.

Table 2: Microbial populations in spoiled pre-formed pizza. Data are the results of the microbiological analysis of 10 samples.

However, Ricciardi et al. [21] observed LAB counts of $10^{7}$ to $10^{8}$ $\mathrm{CFU} \mathrm{g}{ }^{-1}$ in fermented bread, an expected result given that LAB are used to produce this particular fermented product.

The concentration of molds and yeasts ranged from $3.95 \times 10^{4}$ (sample 4) to $9.17 \times 10^{5}$ (sample 8 ) $\mathrm{CFU} \mathrm{g}^{-1}$ in pizza products (Table 2). These counts are comparable with those recorded by Rodríguez et al. [22] of 3.78 to $6.09 \log 10 \mathrm{CFU} \mathrm{g}^{-1}$ in sliced wheat flour bread (with preservative) after 18 days of storage at room temperature. Yeast numbers are particularly dependent on the inoculation ratio of the dough. It was shown by Hammes et al. [23] that regardless of the inoculum's origin, yeasts in dough may originate from the flour or other ingredients in the dough and from the bakery environment.

\section{Fungal diversity}

To our knowledge, this is the first study to investigate the fungal (molds and yeasts) microbiota in packaged precooked ready-to-eat pizza using culture-independent analysis. The near full-length fungal $18 \mathrm{~S}$ rRNA gene $(\approx 1,400 \mathrm{bp})$ was amplified using universal fungal $18 \mathrm{~S}$ primers, cloned, and then sequenced. A total of 28 PCR products from these clones were sent for sequencing and the average length of each sequence was $753 \mathrm{bp}$, all of which sharing 99 to100\% similarity with sequences found in public databases. Sequencing of these clones revealed the presence of at least 5 genera of fungi from 2 phyla (Table 3 ).

Among the 28 clones sequenced, 7 clones (clones 9 to 14, and 26) from 4 samples were closely related to the Penicillium genus (Table 3). Penicillium is a genus of ascomycetous fungi that can be found on foodstuffs, leather, and fabrics. They are of economic importance in the production of antibiotics (e.g. penicillin), organic acids, and cheeses [24]. However, the presence of Penicillium spp. in food is also a spoilage issue. Some species produce toxins and may render food inedible or even dangerous for consumption. Hammes et al. [23] reported that Penicillium spp., such as $P$. notatum, $P$. expansum, and $P$. viridicatum, were the predominant spoilage molds in bakery products with a high water activity ( $>86 \%)$. Nielsen et al. [25] also indicated that Penicillium roqueforti is the major contaminant of rye bread. Rodriguez et al. [22] investigated the effect of modified atmosphere packaging on the shelf life of sliced wheat flour bread and identified Penicillium spp. as the most important spoilage agent in this product.

A total of 6 clones (clones 2, 3, and 15 to 18) from 4 samples were identified as either Saccharomyces cerevisiae or Saccharomyces sp. WW-W23 (Table 3). S. cerevisiae, also known as the baker's yeast, has a long history of use in food processing and production. It has been used for centuries as a leavening agent for bread and as a fermenter of alcoholic beverages. With its prolonged use in industrial applications, this yeast has also been the model for various studies on the principles of microbiology [26]. The presence of S. cerevisiae in the product may be due to contamination of the bakery environment with commercial baker's yeast. The investigation of the microbiota of sourdough bread by culture-dependent and culture-independent methods has revealed that the majority of all yeasts isolated from this product are $S$. cerevisiae [27]. S. cerevisiae has also been found to be dominant among yeasts in studies of microbial characterization of sourdough for sweet baked products [28].

Rhodotorula mucilaginosa was the third most frequently fungus identified in this study, with 5 clones (clones 21 to 25) from 3 samples sharing high similarity (100\%) with this species (Table 3 ). This microorganism is an anamorphic genus of heterobasidiomycetous yeasts, normally found in air and soil. Rhodotorula species can also be isolated from human skin, stool, food and fruits and produce pink, orange and red pigments [29]. Red yeasts are the predominant yeasts recognized in many studies of food products and are primarily members of the genera Rhodotorula, Rhodosporidium and Sporobolomyces [30]. Although most species of the genus Rhodotorula are nonpathogenic, some of them are infectious [31]. Rhodotorula is characterized by the absence of ballistoconidia, no fermentation ability, no starch-like compounds, and no xylose in whole-cell hydrolyzates [32].

Three clones from sample 3 shared $99-100 \%$ similarity with the yeast Monascus fuliginosus (Table 3). Species of this genus are commercially important in the production of various Asian fermented foods, e.g. red-rice and food-colouring pigments [33]. Monascus fuliginosus van Tieghem is a cosmopolitan fungus found in soil, soya, sorghum, tobacco, rice, oat and silage [34].

Clone 27 had a high level of identity (99\%) with Galactomyces geotrichum. Galactomyces is an arthroconidial ascomycetous genus [35,36]. G. geotrichum is considered a plant pathogen and is a common post-harvest fungus disease of citrus known as sour rot [37].

A single clone from sample 1 shared $99 \%$ similarity with Hordeum jubatum (foxtail barley). This plant is a short-lived perennial plant species indigenous to Western North America that has become naturalized in Eastern North America. It is most prevalent in soils with a high water table and high salinity [38]. The presence of Hordeum jubatum in the product can be due to contamination of the wheat, which is the main pizza dough ingredient, with foxtail barley seeds from soil.

\section{Pulsed UV light and microbiological analysis}

As sequences closely related to Penicillium spp. were predominant in the samples analyzed, Penicillium roqueforti was used to estimate the reduction of mold spores on the Potato Dextrose Agar surface following exposure to pulsed UV light. It was found that an increase in treatment time significantly $(\mathrm{p}<0.05)$ enhanced $P$. roquefortii inactivation at all three voltages assessed (Figure 1$)$. There were significant $(p<0.05)$ differences between all treatment times $(1,3,5,7$ and $10 \mathrm{~min})$ when $500 \mathrm{~V}$ was applied. For treatments using $750 \mathrm{~V}$, a significant difference $(p<0.05)$ was demonstrated between 1 to 3 and 5 to $7 \mathrm{~min}$. When $1,000 \mathrm{~V}$ was used, UV exposure times of 1 to 3,3 to 5 and 7 to $10 \mathrm{~min}$ showed a significant effect $(\mathrm{P}<0.05)$ on the inactivation of $P$. roqueforti. 


\begin{tabular}{|c|c|c|c|c|c|c|}
\hline Clone & Sample & Phylum & Closest match & Similarity [\%] & Accession number & $\begin{array}{l}\text { Sequence size of the } \\
\text { clones [bp] }\end{array}$ \\
\hline Clone 1 & 1 & Tracheophyta & Hordeum jubatum & 99 & AF168852 & 781 \\
\hline Clone 2 & 2 & Ascomycota & Saccharomyces cerevisiae & 99 & AY99853.1 & 800 \\
\hline Clone 3 & 2 & Ascomycota & Saccharomyces sp. WW-W23 & 99 & DQ345280.1 & 563 \\
\hline Clone 4 & 3 & Ascomycota & Monascus fuliginosus & 99 & HM188430.1 & 800 \\
\hline Clone 5 & 3 & Ascomycota & Monascus fuliginosus & 100 & HM188430.1 & 734 \\
\hline Clone 6 & 3 & Ascomycota & Monascus fuliginosus & 100 & HM188430.1 & 561 \\
\hline Clone 7 & 4 & & Uncultured fungus clone & 99 & JN054689.1 & 561 \\
\hline Clone 8 & 4 & & Uncultured fungus clone & 99 & JN054689.1 & 849 \\
\hline Clone 9 & 5 & Ascomycota & Penicillium expansum & 99 & JX470345.1 & 759 \\
\hline Clone 10 & 5 & Ascomycota & Penicillium expansum & 100 & $J \times 470345.1$ & 734 \\
\hline Clone 11 & 5 & Ascomycota & Penicillium freii & 99 & JX470344.1 & 816 \\
\hline Clone 12 & 6 & Ascomycota & Penicillium freii & 99 & JX470344.1 & 809 \\
\hline Clone 13 & 7 & Ascomycota & Penicillium sp. HSL & 99 & JX910356.1 & 745 \\
\hline Clone 14 & 7 & Ascomycota & Penicillium sp. HSL & 99 & JX910356.1 & 798 \\
\hline Clone 15 & 7 & Ascomycota & Saccharomyces cerevisiae & 100 & KF447113.1 & 808 \\
\hline Clone 16 & 8 & Ascomycota & Saccharomyces cerevisiae & 99 & KF447113.1 & 828 \\
\hline Clone 17 & 8 & Ascomycota & Saccharomyces cerevisiae & 100 & KF447113.1 & 766 \\
\hline Clone 18 & 5 & Ascomycota & Saccharomyces cerevisiae & 99 & KF447113.1 & 797 \\
\hline Clone 19 & 6 & & Uncultured fungus clone & 99 & KC337083 & 814 \\
\hline Clone 20 & 7 & & Uncultured fungus clone & 99 & KC337083 & 826 \\
\hline Clone 21 & 7 & Basidiomycota & Rhodotorula mucilaginosa & 100 & KC186124 & 803 \\
\hline Clone 22 & 1 & Basidiomycota & Rhodotorula mucilaginosa & 100 & KC186125 & 803 \\
\hline Clone 23 & 1 & Basidiomycota & Rhodotorula mucilaginosa & 100 & KC186126 & 802 \\
\hline Clone 24 & 1 & Basidiomycota & Rhodotorula mucilaginosa & 100 & KC186127 & 803 \\
\hline Clone 25 & 2 & Basidiomycota & Rhodotorula mucilaginosa & 100 & KC186128 & 766 \\
\hline Clone 26 & 2 & Ascomycota & Penicillium sp. ljg1 & 99 & KC833482 & 691 \\
\hline Clone 27 & 4 & Ascomycota & Galactomyces geotrichum & 99 & JQ668740 & 529 \\
\hline Clone 28 & 4 & & Uncultured eukaryote & 99 & JX394808 & 728 \\
\hline
\end{tabular}

Table 3: Tentative identification of clones by sequencing and BLAST analysis. Clone sequences were compared to $16 \mathrm{~S}$ rRNA gene sequences in the GenBank database with BLAST.

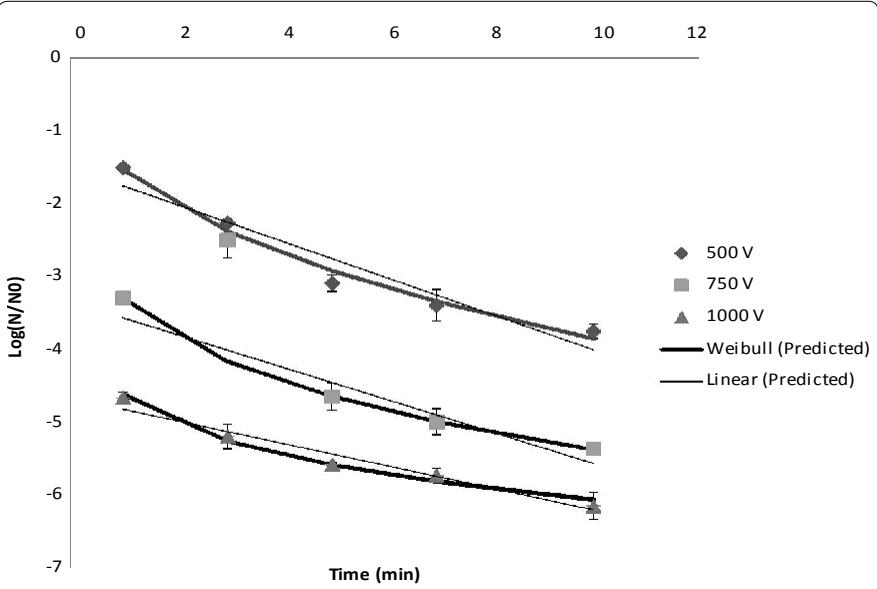

Figure 1: Inactivation of Penicillium roqueforti with pulsed UV light fitted with the first order and Weibull kinetics models. Inactivation was performed on Potato Dextrose Agar at three different voltage inputs $(500,750$, and 1,000 $\mathrm{V})$ and five different treatment times (1, 3, 5, 7, and 10 mins). Data shown are mean and standard deviation values.

Therefore, it can be noted that at a specific energy, the reduction in the population of $P$. roquefort $i$ improved significantly with an increase in the treatment time.

According to our results, there was also a significant $(\mathrm{p}<0.05)$ improvement in the reduction of $P$. roqueforti when this mold was subjected to an increase in the voltage with a constant pulse number (or treatment time). After 10 min of pulsed UV light treatment, a log10 reduction of 3.74, 5.36 and $6.14 \mathrm{CFU} \mathrm{ml}^{-1}$ was obtained using 500, 750 and $1,000 \mathrm{~V}$, respectively. Marquenie et al. [10] also reported a maximal inactivation of 3 and $4 \log 10$ units for conidia of Botrytis cinerea and Monilinia fructigena in vitro after pulse UV treatment of $120 \mathrm{~s}$ at the fluence of $0.10 \mathrm{~J} \mathrm{~cm}^{-2}$. The treated fungal populations of Aspergillus niger and Fusarium culmorum were reduced by 3 to $4.5 \log 10$ orders after 1,000 light pulses of the $3 \mathrm{~J} \mathrm{UV} \mathrm{intensity} \mathrm{light} \mathrm{[26].} \mathrm{The} \mathrm{maximal}$ $\log 10$ reduction was close to 1 with a pulsed UV-light fluence of $1.2 \mathrm{~J}$ $\mathrm{cm}^{-2}$ for Aspergillus niger inoculated in sugar syrup, whereas on agar, the $\log 10$ reduction for the same fungi was even lower than 1 for the same fluence $[39,40]$.

Taking into account that the solid agar used in the experiments was artificially contaminated, the initial number of mold spores was higher than in industrial conditions. Therefore, it could be estimated that it is possible to achieve a complete decontamination by a 6.14 $\log 10 \mathrm{CFU} \mathrm{ml} \mathrm{m}^{-1}$ reduction (the highest mold reduction achieved in this experiment). Therefore, the results of this work show that pulsed light can be considered a promising technique for fungi elimination or decontamination in the bakery industry.

\section{Inactivation kinetics model}

The comparison of the predicted and experimental inactivation data is shown in Figure 1. It was observed that none of the inactivation curves (at different voltages) exhibited a linear trend. As indicated 
by these results, the Weibull model was found to more accurately estimate the microbial reductions obtained during pulsed UV light treatments. Bialka et al. [17] also reported that first-order kinetics are not suitable for the estimation of microbial inactivation on berries treated with ozone or pulsed UV-light, but that the Weibull model can be successfully used to estimate the reductions of E. coli O157:H7 and Salmonella enterica on raspberries and strawberries. A modified Weibull model was chosen by Levy et al. [40] to fit microbial reduction curves for Bacillus subtilis, B. atrophaeus, B. cereus, Geobacillus stearothermophilus, and Aspergillus niger treated by pulsed UV-light on solid agar. Koseki and Yamamoto [41] showed that a linear model was not suitable to describe the reduction of Escherichia coli during high pressure processing. Their findings also demonstrate that the Weibull model as well as the modified Baranyi model could be used to accurately estimate this inactivation and were capable of fitting the tail of the survivor curve resulting from high pressure processing. The ability of this model to accurately estimate reductions of $P$. roquefort $i$ can be seen in Figure 1.

Goodness-of-fit parameters of log-linear and Weibull models for pulsed UV-light treatment are shown in Tables 4 and 5 , respectively. These parameters provide greater insight into the shape of the inactivation curves. Overall, the RMSE and $\mathrm{R}^{2}$ values obtained for the Weibull model are less than and greater than, respectively, those obtained for the log-linear model, with the exception of $750 \mathrm{~V}$ due to the existence of an outliner in the results. The scale parameter $\alpha$ is usually considered as a measure of the organism resistance (kinetic parameter) to treatment and decreases with exposure time. For the Weibull model, this value was found to be decreasing when voltage increased. For the three voltages, $\beta$ values were less than 1 , which accounts for curve's upward concavity, but also indicated that the remaining cells are less susceptible to pulsed UV-light, perhaps due to lack of pulsed light penetrability. A trend can also be seen in the dose required for 1 $\mathrm{CFU} \mathrm{ml}{ }^{-1} \log 10$ reduction (tR): values for tR decrease when a higher voltage is applied, which indicates the cells are inactivated quickly with higher voltages. In fact, pulsed UV light irradiation was very effective at inactivating the spores of $P$. roqueforti; however, the dose of energy per pulse and the position of xenon lamp will determine the lethality. Moreover, decontamination by pulsed UV-light has to account for possible interactions between the target surface and the microorganism. Therefore, further research on the influence of pulsed light on foods with more complex composition is needed to define the applicability of the technology in decontamination processes [42].

Based on culture-dependent analysis, the number of microbes in the precooked pizza samples in decreasing order of concentration was: Mesophilic aerobic bacteria (MAB), molds and yeasts $(\mathrm{M}+\mathrm{Y})$, mesophilic anaerobic bacteria (MANB), and finally lactic acid bacteria (LAB). Analysis of $18 \mathrm{~S}$ rDNA PCR-amplified clones revealed fungi communities with low diversity in ready-to-eat pizza samples. The spoilage microbiota was mostly comprised of fungi with a high sequence similarity to the Penicillium mold genus and to the Saccharomyces and Rhodotorula yeast genera.

The most effective pulsed UV treatment for inactivating $P$. roqueforti was found to be $1,000 \mathrm{~V}$ for $10 \mathrm{~min}$. The experimental data also show that inactivation of $P$. roquefort $i$ by pulsed UV light does not follow first-order kinetics, but that the inactivation kinetics of the tested microorganism on solid agar was best described by the Weibull model with the smallest RMSE and $\mathrm{R}^{2} \geq 0.92$. The results of this work show that pulsed light can be considered a promising technique for fungi elimination or decontamination in the bakery industry.

\begin{tabular}{|c|c|c|c|}
\hline Voltage & RMSE & $\mathbf{R}^{2}$ & K \\
\hline $500 \mathrm{~V}$ & 0.29317 & 0.9219 & 0.2497 \\
\hline $750 \mathrm{~V}$ & 0.72810 & 0.7296 & 0.2965 \\
\hline $1,000 \mathrm{~V}$ & 0.13248 & 0.9584 & 0.9584 \\
\hline
\end{tabular}

RMSE: Root Mean Square Error; $\mathrm{R}^{2}$ : Root square; K: First-order extinction coefficient.

Table 4: Goodness-of-fit parameters of the first order kinetics models estimating reductions of $P$. roqueforti on solid agar after treatment with pulsed UV-light.

\begin{tabular}{|l|l|l|l|l|l|}
\hline Voltage & $\boldsymbol{\alpha}$ & $\boldsymbol{\beta}$ & $\mathbf{t}_{\mathbf{R}}$ & $\mathbf{R M S E}$ & $\mathbf{R}^{2}$ \\
\hline $500 \mathrm{~V}$ & 0.04326 & 0.40072 & 0.34687 & 0.1374 & 0.9828 \\
\hline $750 \mathrm{~V}$ & 0.00260 & 0.30359 & 0.04063 & 0.8163 & 0.6601 \\
\hline $1,000 \mathrm{~V}$ & $2.2 \times 10^{-9}$ & 0.11851 & $2.51 \times 10^{-6}$ & 0.0824 & 0.9839 \\
\hline
\end{tabular}

$\alpha$ : Characteristic time [s or min]; $\beta$ : Shape parameter; $t_{\mathrm{R}}$ : Reliable life [indicating the time necessary for $90 \%$ reduction in the number of microorganism]; RMSE: Root Mean Square Error; $R^{2}$ : Root square

Table 5: Goodness-of-fit parameters of Weibull models estimating reductions of $P$. roqueforti on solid agar after treatment with pulsed UV-light.

\section{Acknowledgements}

This work was supported by Ministère de l'Agriculture, des Pêcheries et de l'Alimentation du Quebec (MAPAQ) through a grant (Programme Innov'Action Agroalimentaire) held by M.O.N. and M.R.C. MAPAQ was not involved in this work.

\section{References}

1. Pinho BH, Furlong EB (2000) The occurrence of molds, yeasts and mycotoxins in pre-cooked pizza dough sold in Southern Rio Grande do Sul. Braz J Microbiol 31: 99-102.

2. De Vuyst L, Vancanneyt M (2007) Biodiversity and identification of sourdough lactic acid bacteria. Food Microbiol 24: 120-127.

3. Scheirlinck I, Van der Meulen R, Van Schoor A, Vancanneyt M, De Vuyst L, et al. (2007) Influence of geographical origin and flour type on diversity of lactic acid bacteria in traditional Belgian sourdoughs. Appl Environ Microbiol 73: 6262-6269.

4. Díez B, Pedrós-Alió C, Massana R (2001) Study of genetic diversity of eukaryotic picoplankton in different oceanic regions by small-subunit rRNA gene cloning and sequencing. Appl Environ Microbiol 67: 2932-2941.

5. Mcdonald KF, Curry RD, Clevenger TE, Unklesbay K, Eisenstark A, et al (2000) A comparison of pulsed and continuous ultraviolet light sources for the decontamination of surfaces. IEEE Trans. Plasma Sci 28: 1581-1587.

6. Ben Saïd NE, Federighi M, Bakhrouf A, Orange N (2010) Effectiveness of pulsed ultraviolet-light treatment for bacterial inactivation on agar surface and liquid medium. Foodborne Pathog Dis 7: 1401-1406.

7. Dunn J, Burgess D, Leo F (1997) Investigation of pulsed light for termina sterilization of WFI filled blow/fill/seal polyethylene containers. PDA J Pharm Sci Technol 51: 111-115.

8. Begum M, Hocking AD, Miskelly D (2009) Inactivation of food spoilage fungi by ultra violet (UVC) irradiation. Int J Food Microbiol 129: 74-77.

9. Hosseini S, Azar-Daryany M, Massudi R, Elikaei A (2011) Pulsed UV laser light on Escherichia coli and Saccharomyces cerevisiae suspended in non-alcoholic beer. Iran J Microbiol 3: 31-35.

10. Marquenie D, Michiels CW, Van Impe JF, Schrevens E, Nicolai BN (2003) Pulsed white light in combination with UV-C and heat to reduce storage rot of strawberry. Postharvest Biol Tec 28: 455-461.

11. Jun S, Irudayaraj J, Demirci A, Geiser D (2003) Pulsed UV-light treatment of corn meal for inactivation of Aspergillus niger spores. Int J Food Sci Techno 38: 883-888.

12. U.S. Environmental Protection Agency (2003) Appendix J. Pilot-scale and demonstration-scale testing. J-4, J-5. Ultraviolet Disinfection Guidance Manual. Washington, DC

13. U.S. Food and Drug Administration (2013) Kinetics of microbial inactivation for alternative food processing technologies: Pulsed light technology. 
14. Terzaghi BE, Sandine WE (1975) Improved medium for lactic streptococci and their bacteriophages. Appl Microbiol 29: 807-813.

15. Wu Z, Wang XR, Blomquist G (2002) Evaluation of PCR primers and PCR conditions for specific detection of common airborne fungi. J Environ Monit 4 377-382.

16. Wright ES, Yilmaz LS, Noguera DR (2012) DECIPHER, a search-based approach to chimera identification for 16S rRNA sequences. Appl Environ Microbiol 78: 717-725.

17. Bialka KL, Demirci A, Puri VM (2008) Modeling the inactivation of Escherichia coli $0157: \mathrm{H} 7$ and Salmonella enterica on raspberries and strawberries resulting from exposure to ozone or pulsed UV-light. J Food Eng 85: 444-449.

18. van Boekel MA (2002) On the use of the Weibull model to describe thermal inactivation of microbial vegetative cells. Int J Food Microbiol 74: 139-159.

19. Unluturk S, Atilgan MR, Baysal AH, Unluturk MS (2010) Modeling inactivation kinetics of liquid egg white exposed to UV-C irradiation. Int J Food Microbiol 142: 341-347.

20. Rodríguez V, Medina L, Jordano R (2003) Influence of modified atmosphere packaging on the shelf life of prebaked pizza dough with and without preservative added. Nahrung 47: 122-125.

21. Ricciardi A, Parente E, Piraino P, Paraggio M, Romano P (2005) Phenotypic characterization of lactic acid bacteria from sourdoughs for Altamura bread produced in Apulia (Southern Italy). Int J Food Microbiol 98: 63-72.

22. Rodríguez M, Medina LM, Jordano R (2000) Effect of modified atmosphere packaging on the shelf life of sliced wheat flour bread. Nahrung 44: 247-252.

23. Hammes WP, Brandt MJ, Francis KL, Rosenheim J, Seitter MFH, et al. (2005) Microbial ecology of cereal fermentations. Trends Food Sci Technol 16: 4-11.

24. Tiwari KL, Jadhav SK, Kumar A (2011) Morphological and molecular study of different Penicillium species. Middle East J Sci Res 7: 203-210.

25. Nielsen PV, Suhr KI (2005) Inhibition of fungal growth on wheat and rye bread by modified atmosphere packaging and active packaging using volatile mustard essential oil. J Food Sci 70: M37-M44.

26. Anderson JG, Rowan NJ, Macgregor SJ, Fouracre RA, Farish O, et al. (2000) Inactivation of food-borne enteropathogenic bacteria and spoilage fungi using pulsed-light. IEEE Trans Plasma Sci 28: 83-88.

27. lacumin L, Cecchini F, Manzano M, Osualdini M, Boscolo D, et al. (2009) Description of the microflora of sourdoughs by culture-dependent and cultureindependent methods. Food Microbiol 26: 128-135.

28. Palomba S, Blaiotta G, Ventorino V, Saccone A, Pepe O (2010) Microbia characterization of sourdough for sweet baked products in the Campania region (Southern Italy) by a polyphasic approach. Ann Microbiol 61: 307-314.

29. Kreger-van Rij NJW (1984) Systems of classification of the yeasts. The Yeasts: A Taxonomic Study. (3rdedn), Elsevier. Amsterdam, The Netherlands.

30. Hagler AN, Ahearn BG (1987) Ecology of aquatic yeasts. Rose AH, Harrison JS (Editors). The yeasts, second edition, vol. 1 (pp. 181-205). Academic Press, London, United Kingdom.

31. Mokhtari M, Etebarian HR, Mirhendi SH, Razavi M (2011) Identification and phylogeny of some species of the genera Sporidiobolus and Rhodotorula using analysis of the $5.8 \mathrm{~S}$ rDNA gene and two ribosomal internal transcribed spacers. Arch Biol Sci 63: 79-88.

32. Nagahama T, Hamamoto M, Nakase T, Takami H, Horikoshi K (2001) Distribution and identification of red yeasts in deep-sea environments around the northwest Pacific Ocean. Antonie Van Leeuwenhoek 80: 101-110.

33. Steinkraus KH (1984) Handbook of Indigenous Fermented Foods. Dekker. New York, USA.

34. Messing J, Vieira J, (1982) A new pair of M13 vectors for selecting either DNA strand of double-digest restriction fragments. Gene 19: 269-276.

35. Kurtzman CP, Robnett CJ (1998) Identification and phylogeny of ascomycetous yeasts from analysis of nuclear large subunit (26S) ribosomal DNA partia sequences. Antonie Van Leeuwenhoek 73: 331-371.

36. Ueda-Nishimura K, Mikata K (2000) Two distinct 18S rRNA secondary structures in Dipodascus (Hemiascomycetes). Microbiology 146 : 1045-1051.

37. Pimenta RS, Alves PD, Corrêa A Jr, Lachance MA, Prasad GS, et al. (2005) Geotrichum silvicola sp. nov., a novel asexual arthroconidial yeast species related to the genus Galactomyces. Int J Syst Evol Microbiol 55: 497-501.

38. Badger KS, Ungar IA (1989) The effects of salinity and temperature on the germination of the inland halophyte Hordeum jubatum. Can J Bot 67: 14201425

39. Chaine A, Levy C, Lacour B, Riedel C, Carlin F (2012) Decontamination of sugar syrup by pulsed light. J Food Prot 75: 913-917.

40. Levy C, Aubert X, Lacour B, Carlin F (2012) Relevant factors affecting microbial surface decontamination by pulsed light. Int J Food Microbiol 152: 168-174.

41. Koseki S, Yamamoto K (2007) A novel approach to predicting microbial inactivation kinetics during high pressure processing. Int J Food Microbiol 116 275-282.

42. Moharram AM, Mostafa EM, Ismail MA (2011) Chemical profile of Monascus ruber strains. Food Technol Biotech 50: 490-499. 